\title{
Permutation statistics and multiple pattern avoidance
}

\author{
WuTTISAK TRONGSIRIWAT
}

\begin{abstract}
For a set of patterns $\Pi$, let $F_{n}^{\text {st }}(\Pi ; q)$ be the st-polynomial of permutations avoiding all patterns in $\Pi$. Suppose $312 \in \Pi$. For some permutation statistic st, we give a formula that expresses $F_{n}^{\text {st }}(\Pi ; q)$ in terms of these st-polynomials where we take some subblocks of the patterns in $\Pi$. Using this formula, we construct examples of nontrivial st-Wilf equivalences. In particular, this disproves a conjecture by Dokos, Dwyer, Johnson, Sagan, and Selsor that all inv-Wilf equivalences are trivial.
\end{abstract}

\section{Introduction}

Let $\mathfrak{S}_{n}$ be the set of permutations of $[n]:=\{1,2, \ldots, n\}$ and let $\mathfrak{S}=$ $\bigcup_{n \geq 0} \mathfrak{S}_{n}$, where $\mathfrak{S}_{0}$ contains only one element $\epsilon$ - the empty permutation. For permutations $\pi, \sigma \in \mathfrak{S}$ we say that the permutation $\sigma$ contains $\pi$ if there is a subsequence of $\sigma$ having the same relative order as $\pi$. In particular, every permutation contains $\epsilon$, and every permutation except $\epsilon$ contains $1 \in \mathfrak{S}_{1}$. For consistency, we will use the letter $\sigma$ to represent a permutation and $\pi$ to represent a pattern. We say that $\sigma$ avoids $\pi$ (or $\sigma$ is $\pi$-avoiding) if $\sigma$ does not contain $\pi$. For example, the permutation 46127538 contains 3142 while the permutation 46123578 avoids 3142 . We denote by $\mathfrak{S}_{n}(\pi)$, where $\pi \in \mathfrak{S}$, the set of permutations $\sigma \in \mathfrak{S}_{n}$ avoiding $\pi$. More generally we denote by $\mathfrak{S}_{n}(\Pi)$, where $\Pi \subseteq \mathfrak{S}$, the set of permutations avoiding each pattern $\pi \in \Pi$ simultaneously, i.e. $\mathfrak{S}_{n}(\Pi)=\bigcap_{\pi \in \Pi} \mathfrak{S}_{n}(\pi)$. Two sets of patterns $\Pi$ and $\Pi^{\prime}$ are called Wilf equivalent, written $\Pi \equiv \Pi^{\prime}$, if $\left|\mathfrak{S}_{n}(\Pi)\right|=\left|\mathfrak{S}_{n}\left(\Pi^{\prime}\right)\right|$ for all integers $n \geq 0$.

Now we define $q$-analogues of pattern avoidance using permutation statistics. A permutation statistic (or sometimes just statistic) is a function st: $\mathfrak{S} \rightarrow \mathbb{N}$, where $\mathbb{N}$ is the set of nonnegative integers. Given a permutation statistic st, we define the st-polynomial of $\Pi$-avoiding permutations to be

$$
F_{n}^{\mathrm{st}}(\Pi)=F_{n}^{\mathrm{st}}(\Pi ; q):=\sum_{\sigma \in \mathfrak{S}_{n}(\Pi)} q^{\mathrm{st}(\sigma)} .
$$

arXiv: 1309.3028 
We may drop the $q$ if it is clear from the context. The sets of patterns $\Pi$ and $\Pi^{\prime}$ are said to be st-Wilf equivalent, written $\Pi \stackrel{\text { st }}{=} \Pi^{\prime}$, if $F_{n}^{\mathrm{st}}(\Pi ; q)=F_{n}^{\mathrm{st}}\left(\Pi^{\prime} ; q\right)$ for all $n \geq 0$.

The study of $q$-analogues of pattern avoidance using permutation statistics and the st-Wilf equivalences began in 2002, as initiated by Robertson, Saracino, and Zeilberger [6], with the emphasis on the number of fixed points. Elizalde subsequently refined results of Robertson et al. by considering the excedance statistic [2] and later extended the study to cases of multiple patterns [3]. A bijective proof was later given by Elizalde and Pak [4]. Dokos et al. [1] studied pattern avoidance on the inversion and major statistics, as remarked by Savage and Sagan in their study of Mahonian pairs [7].

In this paper, we study multiple pattern avoidance on a class of permutation statistics which includes the inversion and descent statistics. The inversion number of $\sigma \in \mathfrak{S}_{n}$ is

$$
\operatorname{inv}(\sigma)=\#\left\{(i, j) \in[n]^{2}: i<j \text { and } \sigma(i)>\sigma(j)\right\}
$$

The descent number of $\sigma \in \mathfrak{S}_{n}$ is

$$
\operatorname{des}(\sigma)=\#\{i \in[n-1]: \sigma(i)>\sigma(i+1)\}
$$

For example $\operatorname{inv}(3142)=\#\{(1,2),(1,4),(3,4)\}=3$ and $\operatorname{des}(3142)=\#\{1,3\}$ $=2$.

In [1], Dokos et al. conjectured that there are only essentially trivial inv-Wilf equivalences, obtained by rotations and reflections of permutation matrices. Let us describe these more precisely. The notations used below are mostly taken from [1].

Given a permutation $\sigma \in \mathfrak{S}_{n}$, we represent it geometrically using the squares $(1, \sigma(1)),(2, \sigma(2)), \ldots,(n, \sigma(n))$ of the $n$-by- $n$ grid, which is coordinated according to the $x y$-plane. This will be referred as the permutation matrix of $\sigma$. The diagram to the left in Figure 1 is the permutation matrix of 46127538. In the diagram to the right, the red squares correspond to the subsequence 4173, which is an occurrence of the pattern 3142 .

By representing each $\sigma \in \mathfrak{S}$ as a permutation matrix, we have an action of the dihedral group of square $D_{4}$ on $\mathfrak{S}$ by the corresponding action on the permutation matrices. We denote the elements of $D_{4}$ by

$$
D_{4}=\left\{R_{0}, R_{90}, R_{180}, R_{270}, r_{-1}, r_{0}, r_{1}, r_{\infty}\right\}
$$

where $R_{\theta}$ is the counterclockwise rotation by $\theta$ degrees and $r_{m}$ is the reflection in a line of slope $m$. We will sometimes write $\Pi^{t}$ for $r_{-1}(\Pi)$. Note that 

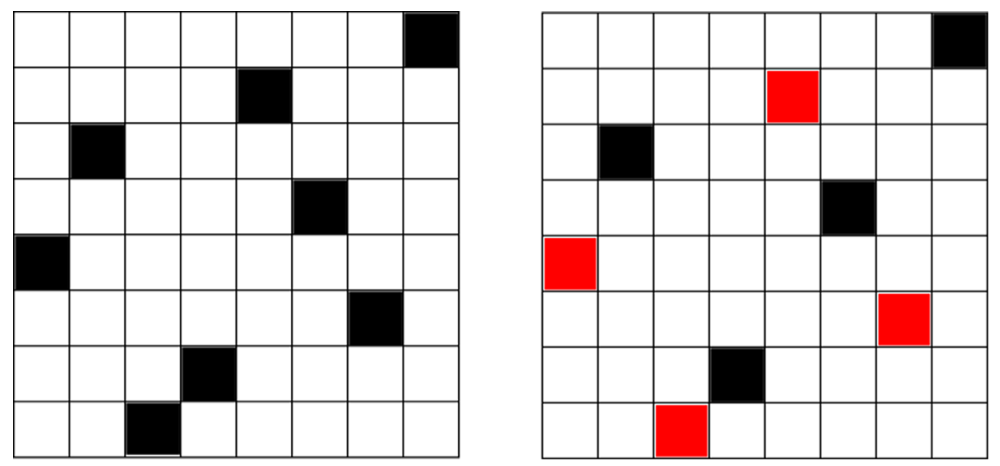

Figure 1: The permutation matrix of 46127538 (left) with an occurrence of 3142 colored (right).

$R_{0}, R_{180}, r_{-1}$, and $r_{1}$ preserve the inversion statistic while the others reverse it, i.e.

$$
\operatorname{inv}(f(\sigma))= \begin{cases}\operatorname{inv}(\sigma) & \text { if } f \in\left\{R_{0}, R_{180}, r_{-1}, r_{1}\right\}, \\
\left(\begin{array}{l}
n \\
2
\end{array}\right)-\operatorname{inv}(\sigma) & \text { if } f \in\left\{R_{90}, R_{270}, r_{0}, r_{\infty}\right\} .\end{cases}
$$

It follows that $\Pi$ and $f(\Pi)$ are inv-Wilf equivalent for all $\Pi \subseteq \mathfrak{S}$ and $f \in$ $\left\{R_{0}, R_{180}, r_{-1}, r_{1}\right\}$. We call these equivalences trivial. With these notations, the conjecture by Dokos et al. can be stated as the following.

Conjecture 1.1 ([1], conj. 2.4). $\Pi$ and $\Pi^{\prime}$ are inv-Wilf equivalent iff $\Pi=$ $f\left(\Pi^{\prime}\right)$ for some $f \in\left\{R_{0}, R_{180}, r_{-1}, r_{1}\right\}$.

Given permutations $\pi=a_{1} a_{2} \ldots a_{k} \in \mathfrak{S}_{k}$ and $\sigma_{1}, \ldots, \sigma_{k} \in \mathfrak{S}$, the $i n$ flation $\pi\left[\sigma_{1}, \ldots, \sigma_{k}\right]$ of $\pi$ by the $\sigma_{i}$ is the permutation whose permutation matrix is obtained by putting the permutation matrices of $\sigma_{i}$ in the relative order of $\pi$; for instance, 213[123,1,21] $=234165$ as illustrated in Figure 2.

For convenience, we write

$$
\pi_{*}:=21[\pi, 1] .
$$

In other words, $\pi_{*}$ is the permutation whose permutation matrix is obtained by adding a box to the lower right corner of the permutation matrix of $\pi$.

The next proposition is one of the main results of this paper, which disproves the conjecture above. This is a special case of the corollary of Theorem 2.4 in the next section.

Proposition 1.2. Let $\iota_{r}$ be the permutation $12 \ldots r \in \mathfrak{S}_{r}$. Let $\pi_{1}, \ldots, \pi_{r}, \pi_{1}^{\prime}$, $\ldots, \pi_{r}^{\prime}$ be permutations such that $\left\{312, \pi_{i}\right\} \stackrel{i n v}{=}\left\{312, \pi_{i}^{\prime}\right\}$ for all $i$. Set $\pi=$ 


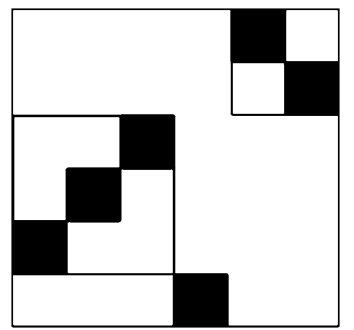

Figure 2: The permutation 213[123,1,21].

$\iota_{r}\left[\pi_{1 *}, \ldots, \pi_{r *}\right]$ and $\pi^{\prime}=\iota_{r}\left[\pi_{1 *}^{\prime}, \ldots, \pi_{r *}^{\prime}\right]$. Then $\{312, \pi\}$ and $\left\{312, \pi^{\prime}\right\}$ are inv-Wilf equivalent, i.e. $F_{n}^{\text {inv }}(312, \pi)=F_{n}^{i n v}\left(312, \pi^{\prime}\right)$ for all $n$.

In particular, if we set each $\pi_{i}^{\prime}$ to be either $\pi_{i}$ or $\pi_{i}^{t}$, then the conditions $\left\{312, \pi_{i}\right\} \stackrel{\text { inv }}{\equiv}\left\{312, \pi_{i}^{\prime}\right\}$ are satisfied. By this construction $\Pi^{\prime}$ is generally not of the form $f(\Pi)$ for any $f \in\left\{R_{0}, R_{180}, r_{-1}, r_{1}\right\}$. For example, the pair $\Pi=\{312,32415\}$ and $\Pi^{\prime}=\{312,24315\}$ is an example of smallest size of nontrivial inv-Wilf equivalences constructed this way.

\section{Avoiding two patterns}

In this section, we study the st-polynomials in the case when $\Pi$ consists of 312 and another permutation $\pi$. For this set of patterns $\Pi$, Mansour and Vainshtein [5] gave a recursive formula for $\left|\mathfrak{S}_{n}(\Pi)\right|$. Here, we give a recursive formula for the st-polynomials $F_{n}^{\text {st }}(\Pi)$, which generalizes the result of Mansour and Vainshtein. Then we present its corollary, which gives a construction of nontrivial st-Wilf equivalences. We note that Proposition 2.1 and Lemma 2.2 appear in [5] as small observations.

Suppose $\sigma \in \mathfrak{S}_{n+1}(312)$ with $\sigma(k+1)=1$. Then, for every pair of indices $(i, j)$ with $i<k+1<j$, we must have $\sigma(i)<\sigma(j)$; otherwise $\sigma(i) \sigma(k+1) \sigma(j)$ is an occurrence of the pattern 312 in $\sigma$. So $\sigma$ can be written as $\sigma=213\left[\sigma_{1}, 1, \sigma_{2}\right]$ with $\sigma_{1} \in \mathfrak{S}_{k}$ and $\sigma_{2} \in \mathfrak{S}_{n-k}$. For the rest of the paper, we will always consider $\sigma$ in this inflation form.

We also assume that the permutation statistic st $: \mathfrak{S}_{n} \rightarrow \mathbb{N}$ satisfies

$$
\operatorname{st}(\sigma)=f(k, n-k)+\operatorname{st}\left(\sigma_{1}\right)+\operatorname{st}\left(\sigma_{2}\right)
$$

for some function $f: \mathbb{N}^{2} \rightarrow \mathbb{N}$ which does not depend on $\sigma$. Some examples of such statistics are the inversion number, the descent number, and the 
number of occurrences of the consecutive pattern 213:

$$
\underline{213}(\sigma)=\#\{i \in[n-2]: \sigma(i+1)<\sigma(i)<\sigma(i+2)\}
$$

For these statistics, we have

$$
\begin{aligned}
& \operatorname{inv}(\sigma)=k+\operatorname{inv}\left(\sigma_{1}\right)+\operatorname{inv}\left(\sigma_{2}\right), \\
& \operatorname{des}(\sigma)=1-\delta_{0, k}+\operatorname{des}\left(\sigma_{1}\right)+\operatorname{des}\left(\sigma_{2}\right), \\
& \underline{213}(\sigma)=\left(1-\delta_{0, k}\right)\left(1-\delta_{k, n}\right)+\underline{213}\left(\sigma_{1}\right)+\underline{213}\left(\sigma_{2}\right),
\end{aligned}
$$

where $\delta$ is the Kronecker delta function.

It will be more beneficial to consider the permutation patterns in their block decomposition form as in the following proposition.

Proposition 2.1. Every 312-avoiding permutation $\pi \in \mathfrak{S}_{n}(312)$ can be written uniquely as

$$
\pi=\iota_{r}\left[\pi_{1 *}, \ldots, \pi_{r *}\right]
$$

where $r \geq 0$ and $\pi_{i} \in \mathfrak{S}(312)$.

Proof. The uniqueness part is trivial. The proof of existence of $\pi_{1}, \ldots, \pi_{r}$ is by induction on $n$. If $n=0$, there is nothing to prove. Suppose the result holds for $n$. Suppose that $\pi(k+1)=1$. Then $\pi=213\left[\pi_{1}, 1, \pi^{\prime}\right]=12\left[\pi_{1 *}, \pi^{\prime}\right]$ where $\pi_{1} \in \mathfrak{S}_{k}(312)$ and $\pi^{\prime} \in \mathfrak{S}_{n-k}(312)$. Applying the inductive hypothesis on $\pi^{\prime}$, we are done.

Suppose that $\pi \in \mathfrak{S}_{n}(312)$ has the block decomposition $\pi=\iota_{r}\left[\pi_{1 *}, \ldots\right.$, $\left.\pi_{r *}\right]$. For $1 \leq i \leq r$, we define $\underline{\pi}(i)$ and $\bar{\pi}(i)$ to be

$$
\underline{\pi}(i)= \begin{cases}\pi_{1} & \text { if } i=1 \\ \iota_{i}\left[\pi_{1 *}, \ldots, \pi_{i *}\right] & \text { otherwise }\end{cases}
$$

and

$$
\bar{\pi}(i)=\iota_{r-i+1}\left[\pi_{i_{*}}, \ldots, \pi_{r_{*}}\right] .
$$

Let $\Pi$ be a set of patterns containing 312. If $\pi \in \Pi \backslash\{312\}$ contains the pattern 312 , then every permutation avoiding 312 will automatically avoid $\pi$, which means $F_{n}^{\text {st }}(\Pi)=F_{n}^{\text {st }}(\Pi \backslash\{\pi\})$. So we may assume that each pattern besides 312 in $\Pi$ avoids 312 . The following lemma gives a recursive condition for a permutation $\sigma=213\left[\sigma_{1}, 1, \sigma_{2}\right] \in \mathfrak{S}(312)$ to avoid $\pi$, in terms of $\sigma_{1}, \sigma_{2}$, and the blocks $\pi_{i *}$ of $\pi$. 


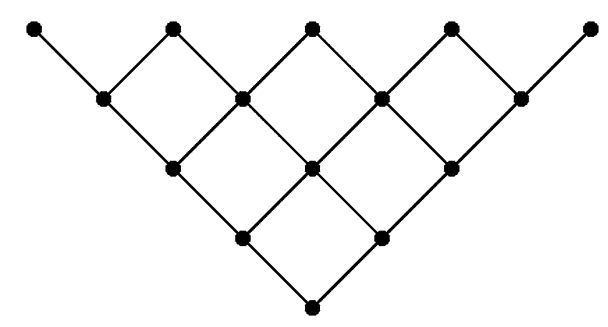

Figure 3: The poset $L_{5}$.

Lemma 2.2. Let $\sigma=213\left[\sigma_{1}, 1, \sigma_{2}\right], \pi=\iota_{r}\left[\pi_{1 *}, \ldots, \pi_{r *}\right] \in \mathfrak{S}(312)$. Then $\sigma$ avoids $\pi$ if and only if the condition

$\left(C_{i}\right): \sigma_{1}$ avoids $\underline{\pi}(i)$ and $\sigma_{2}$ avoids $\bar{\pi}(i)$

holds for some $i \in[r]$.

Proof. First, suppose that $\sigma$ contains $\pi$. Let $j$ be the largest number for which $\sigma_{1}$ contains $\underline{\pi}(j)$. Then $\sigma_{2}$ must contain $\bar{\pi}(j+1)$. So $\sigma_{1}$ contains $\underline{\pi}(i)$ for all $i \leq j$, and $\sigma_{2}$ contains $\bar{\pi}(i)$ for all $i>j$. Thus none of the $C_{i}$ holds.

On the other hand, suppose that there is a permutation $\sigma \in \mathfrak{S}(312)$ that avoids $\pi$ but does not satisfy any $C_{i}$. This means, for every $i$, either $\sigma_{1}$ contains $\underline{\pi}(i)$ or $\sigma_{2}$ contains $\bar{\pi}(i)$. Let $j$ be the smallest number such that $\sigma_{1}$ does not contain $\underline{\pi}(j)$. Note that $j$ exists and $j>1$ since $j=1$ implies $\sigma_{2}$ contains $\bar{\pi}(1)=\pi$, a contradiction. Since $\sigma_{1}$ does not contain $\underline{\pi}(j), \sigma_{2}$ must contain $\bar{\pi}(j)$ (by $C_{j}$ ). But since $\sigma_{1}$ contains $\underline{\pi}(j-1)$ by minimality of $j$, we have found a copy of $\pi$ in $\sigma$ with $\underline{\pi}(j-1)$ from $\sigma_{1}$ and $\bar{\pi}(j)$ from $\sigma_{2}$, a contradiction. (For $j=2$, the number 1 in $\sigma$ together with $\pi_{1}$ in $\sigma_{1}$ give $\left.\pi_{1 *}.\right)$

Before presenting the main result, we state a technical lemma regarding the Möbius function of certain posets. See, for example, Chapter 3 of [8] for definitions and terminologies about posets and the general treatment of the subject.

Let $\mathbf{r}$ be the chain of $r$ elements $0<1<\cdots<r-1$. Let $L_{r}$ be the poset obtained by taking the elements of $\mathbf{r} \times \mathbf{r}$ of rank 0 to $r-1$, i.e. the elements of $L_{r}$ are the lattice points $(a, b)$ where $a, b \geq 0$ and $a+b<r$. For instance, $L_{5}$ is the poset shown in Figure 3. We denote its unique minimal element $(0,0)$ by $\hat{0}$. Let $\hat{L}_{r}$ be the poset $L_{r}$ with the unique maximum element $\hat{1}$ adjoined.

For a poset $P$, we denote the Möbius function of $P$ by $\mu_{P}$. Note that for every element $a \in \hat{L}_{r}$ the up-set $U(a):=\left\{x \in \hat{L}_{r}: x \geq a\right\}$ of $a$ is 
isomorphic to $\hat{L}_{r-l(a)}$ where $l(a)$ is the rank of $a$ in $L_{r}$. Therefore, the problem of computing $\mu_{\hat{L}_{r}}(x, \hat{1})$ for every $r$ is equivalent to computing $\mu_{\hat{L}_{r}}(\hat{0}, \hat{1})$ for every $r$, which is given by the following lemma. The proof is omitted since it is by a straightforward calculation.

Lemma 2.3. We have

$$
\mu_{\hat{L}_{r}}(\hat{0}, \hat{1})= \begin{cases}(-1)^{r}, & \text { if } r=1,2, \\ 0, & \text { otherwise }\end{cases}
$$

We now present the main theorem of this section.

Theorem 2.4. Let $\Pi=\{312, \pi\}$. Suppose that the statistic st $: \mathfrak{S} \rightarrow \mathbb{N}$ satisfies the condition ( $\dagger)$. Then $F_{n}^{s t}(\Pi ; q)$ satisfies

$$
\begin{aligned}
F_{n+1}^{s t}(\Pi ; q)=\sum_{k=0}^{n} q^{f(k, n-k)}[ & {\left[\sum_{i=1}^{r} F_{k}^{s t}(312, \underline{\pi}(i)) \cdot F_{n-k}^{s t}(312, \bar{\pi}(i))\right.} \\
(*) & \left.-\sum_{i=1}^{r-1} F_{k}^{s t}(312, \underline{\pi}(i)) \cdot F_{n-k}^{s t}(312, \bar{\pi}(i+1))\right],
\end{aligned}
$$

for all $n \geq 0$, where $F_{0}^{s t}(\Pi ; q)=0$ if $\pi=\epsilon$, and 1 otherwise.

Proof. For $k \in\{0,1, \ldots, n\}$ and $\Sigma \subset \mathfrak{S}$, we write $\mathfrak{S}_{n+1}^{k}(\Sigma)$ to denote the set of permutations $\sigma \in \mathfrak{S}_{n+1}(\Sigma)$ such that $\sigma(k+1)=1$. In particular,

$$
\mathfrak{S}_{n+1}^{k}(312)=\left\{\sigma=213\left[\sigma_{1}, 1, \sigma_{2}\right]: \sigma_{1} \in \mathfrak{S}_{k}(312) \text { and } \sigma_{2} \in \mathfrak{S}_{n-k}(312)\right\}
$$

Fix $k$, and let $A_{i}(i \in[r])$ be the set of permutations in $\mathfrak{S}_{n+1}^{k}(312)$ satisfying the condition $C_{i}$. So $\mathfrak{S}_{n+1}^{k}(\Pi)=A_{1} \cup A_{2} \cup \cdots \cup A_{r}=: A$ by Lemma 2.2 . Observe that if $i_{1}<\cdots<i_{k}$ then

$$
A_{i_{1}} \cap A_{i_{2}} \cap \cdots \cap A_{i_{k}}=A_{i_{1}} \cap A_{i_{k}}=: A_{i_{1}, i_{k}}
$$

since satisfying the conditions $C_{i_{1}}, \ldots, C_{i_{k}}$ is equivalent to satisfying the conditions $C_{i_{1}}$ and $C_{i_{k}}$.

Let $P$ be the intersection poset of $A_{1}, \ldots, A_{r}$, where the order is given by $A \leq B$ if $A \subseteq B$. The elements of $P$ are $A, A_{i}(1 \leq i \leq r)$, and $A_{i, j}$ $(1 \leq i<j \leq r)$. We see that $P$ is isomorphic to the set $\hat{L}_{r}$, so the Möbius 
function $\mu_{P}(T, A)$ for $T \in P$ is given by

$$
\mu_{P}(T, A)= \begin{cases}1 & \text { if } T=A \text { or } A_{i, i+1} \text { for some } i \\ -1 & \text { if } T=A_{i} \text { for some } i \\ 0 & \text { otherwise }\end{cases}
$$

For $T \in P$, we define $g: P \rightarrow \mathbb{C}(x: x \in A)$ by

$$
g(T)=\sum_{x \in T} x
$$

The Möbius inversion formula ([8], Section 3.7) implies that

$$
\begin{aligned}
g(A) & =-\sum_{T<A} \mu_{P}(T, A) g(T) \\
& =\sum_{i=1}^{r} g\left(A_{i}\right)-\sum_{i=1}^{r-1} g\left(A_{i} \cap A_{i+1}\right) .
\end{aligned}
$$

By mapping $\sigma \mapsto q^{\text {st( } \sigma)}$ for all $\sigma \in A, g(A)$ is sent to $F_{n+1, k}^{\text {st }}(\Pi ; q):=$ $\sum_{\sigma \in \mathfrak{S}_{n+1}^{k}(\Pi)} q^{\mathrm{st}(\sigma)}$. Hence,

$$
\begin{aligned}
F_{n+1, k}^{\mathrm{st}}(\Pi ; q) & =\sum_{i=1}^{r} \sum_{\sigma \in A_{i}} q^{\mathrm{st}(\sigma)}-\sum_{i=1}^{r-1} \sum_{\sigma \in A_{i} \cap A_{i+1}} q^{\mathrm{st}(\sigma)} \\
& =q^{f(k, n-k)}\left[\sum_{i=1}^{r} \sum_{\sigma \in A_{i}} q^{\mathrm{st}\left(\sigma_{1}\right)+\operatorname{st}\left(\sigma_{2}\right)}-\sum_{i=1}^{r-1} \sum_{\sigma \in A_{i} \cap A_{i+1}} q^{\mathrm{st}\left(\sigma_{1}\right)+\operatorname{st}\left(\sigma_{2}\right)}\right]
\end{aligned}
$$

where the second equality is obtained from the condition $(\dagger)$.

Note that $\sigma \in A_{i}$ iff $\sigma_{1}$ avoids $\underline{\pi}(i)$ and $\sigma_{2}$ avoids $\bar{\pi}(i)$, and $\sigma \in A_{i} \cap A_{i+1}$ iff $\sigma_{1}$ avoids $\underline{\pi}(i)$ and $\sigma_{2}$ avoids $\bar{\pi}(i+1)$. Thus

$$
\sum_{\sigma \in A_{i}} q^{\mathrm{st}\left(\sigma_{1}\right)+\mathrm{st}\left(\sigma_{2}\right)}=F_{k}^{\mathrm{st}}(312, \underline{\pi}(i)) \cdot F_{n-k}^{\mathrm{st}}(312, \bar{\pi}(i))
$$

and

$$
\sum_{\sigma \in A_{i} \cap A_{i+1}} q^{\mathrm{st}\left(\sigma_{1}\right)+\mathrm{st}\left(\sigma_{2}\right)}=F_{k}^{\mathrm{st}}(312, \underline{\pi}(i)) \cdot F_{n-k}^{\mathrm{st}}(312, \bar{\pi}(i+1)) .
$$


Therefore

$$
\begin{aligned}
F_{n+1, k}^{\mathrm{st}}(\Pi ; q)=q^{f(k, n-k)}[ & \sum_{i=1}^{r} F_{k}^{\mathrm{st}}(312, \underline{\pi}(i)) \cdot F_{n-k}^{\mathrm{st}}(312, \bar{\pi}(i)) \\
& \left.\quad-\sum_{i=1}^{r-1} F_{k}^{\mathrm{st}}(312, \underline{\pi}(i)) \cdot F_{n-k}^{\mathrm{st}}(312, \bar{\pi}(i+1))\right] .
\end{aligned}
$$

We get the stated result by summing the preceding equation from $k=0$ to $n$.

Example 2.5 ( $q$-analogues of odd Fibonacci numbers). It is well known that the permutations avoiding 312 and 1432 are counted by the Fibonacci numbers $F_{2 n+1}$, assuming $F_{1}=F_{2}=1$ (see [9] for example). Let $A_{n}=$ $F_{2 n+1}$. It can be shown that the $A_{n}$ satisfy

$$
A_{n+1}=A_{n}+\sum_{k=0}^{n-1} 2^{n-k-1} A_{k} .
$$

Theorem 2.4 gives $q$-analogues of this relation. Here, we consider the inversion statistic.

Let $\pi=1432=12\left[\epsilon_{*}, 21_{*}\right]$ and $\Pi=\{312, \pi\}$. Since $\underline{\pi}(1)=\epsilon$ and $F_{n}^{\text {inv }}(312, \epsilon)=0$ for all $n$, Theorem 2.4 implies

$$
\begin{aligned}
F_{n+1}^{\mathrm{inv}}(\Pi) & =\sum_{k=0}^{n} F_{k}^{\mathrm{inv}}(\Pi) F_{n-k}^{\mathrm{inv}}(312,321) \\
& =q^{n} F_{n}^{\mathrm{inv}}(\Pi)+\sum_{k=0}^{n-1} q^{k}(1+q)^{n-k-1} F_{k}^{\mathrm{inv}}(\Pi),
\end{aligned}
$$

where the last equality is by [1], Proposition 4.2 .

Corollary 2.6. Let st be a statistic satisfying $(\dagger)$. Let $\pi_{1}, \ldots, \pi_{r}, \pi_{1}^{\prime}, \ldots, \pi_{r}^{\prime}$ be permutations such that $\left\{312, \pi_{i}\right\} \stackrel{\text { st }}{\equiv}\left\{312, \pi_{i}^{\prime}\right\}$ for all $i$. Set $\pi=\iota_{r}\left[\pi_{1 *}, \ldots\right.$, $\left.\pi_{r *}\right]$ and $\pi^{\prime}=\iota_{r}\left[\pi_{1 *}^{\prime}, \ldots, \pi_{r *}^{\prime}\right]$. Then $\{312, \pi\}$ and $\left\{312, \pi^{\prime}\right\}$ are also st-Wilf equivalent, i.e. $F_{n}^{s t}(312, \pi)=F_{n}^{s t}\left(312, \pi^{\prime}\right)$ for all $n$.

Proof. The proof is by induction on $n$. If $n=0$, then the statement trivially holds. Now suppose the statement holds up to $n$. Then for $0 \leq k \leq n$ and $1 \leq i \leq r$, we have $F_{k}^{\mathrm{st}}(312, \underline{\pi}(i))=F_{k}^{\mathrm{st}}\left(312, \underline{\pi}^{\prime}(i)\right)$ and $F_{n-k}^{\mathrm{st}}(312, \bar{\pi}(i))=$ $F_{n-k}^{\text {st }}\left(312, \overline{\pi^{\prime}}(i)\right)$. Hence $F_{n+1}^{\text {st }}(312, \pi)=F_{n+1}^{\text {st }}\left(312, \pi^{\prime}\right)$ by comparing the terms on the right-hand side of $(*)$. 
As mentioned at the end of Section 1, for the inversion statistic we can choose each $\pi_{i}^{\prime}$ to be either $\pi_{i}$ or $\pi_{i}^{t}$. Of course, this construction works for every statistic st satisfying $(\dagger)$ and that $\operatorname{st}(\sigma)=\operatorname{st}\left(\sigma^{t}\right)$ for all $\sigma \in \mathfrak{S}(312)$. Besides the inversion statistic, the descent statistic for example also possesses this property. To justify this fact, we write $\sigma=213\left[\sigma_{1}, 1, \sigma_{2}\right] \in \mathfrak{S}(312)$ where $\sigma_{1}, \sigma_{2} \in \mathfrak{S}$. Observe that $\sigma^{t}=132\left[\sigma_{2}^{t}, \sigma_{1}^{t}, 1\right]$ and

$$
\operatorname{des}\left(\sigma^{t}\right)=\operatorname{des}\left(\sigma_{2}^{t}\right)+\operatorname{des}\left(\sigma_{1}^{t}\right)+\left(1-\delta_{0, k}\right)
$$

where $k=\left|\sigma_{1}^{t}\right|=\left|\sigma_{1}\right|$. The proof then proceeds by induction on $n=|\sigma|$. It is, however, not true in general that the matrix transposition preserves the descent number. For instance, if $\sigma=2413$, then $\operatorname{des}(\sigma)=1$ while $\operatorname{des}\left(\sigma^{t}\right)=2$.

\section{Generalization}

In this section, we generalize the results from Section 2 to the case when $\Pi$ consists of 312 and other patterns. We again begin with a lemma regarding the Möbius function.

Lemma 3.1. Let $L$ be the poset $L_{r_{1}} \times \cdots \times L_{r_{m}}$ and $\hat{L}$ the poset $L \cup\{\hat{1}\}$. Let $\mu=\mu_{\hat{L}}$ be the Möbius function on $\hat{L}$. Then $\mu(\hat{0}, \hat{1})=0$ unless each $r_{i} \in\{1,2\}$, in which case $\mu(\hat{0}, \hat{1})=(-1)^{|S|+1}$, where $S=\left\{i: r_{i}=2\right\}$.

Proof. Let $a=\left(a_{1}, \ldots, a_{m}\right) \in L$. Then $\mu(\hat{0}, a)=\prod_{i=1}^{m} \mu_{i}\left(\hat{0}, a_{i}\right)$, where $\mu_{i}$ is the Möbius function of $L_{r_{i}}$. So

$$
\mu(\hat{0}, \hat{1})=-\sum_{a \in L} \mu(\hat{0}, a)=-\prod_{i=1}^{m}\left(\sum_{a_{i} \in L_{r_{i}}} \mu_{i}\left(\hat{0}, a_{i}\right)\right)
$$

Note that if $r \geq 3$, the Möbius function $\mu_{L_{r}}(\hat{0}, a)$ vanishes unless $a \in$ $\{(0,0),(1,0),(0, \overline{1}),(1,1)\}$, in which cases the value of $\mu_{L_{r}}(\hat{0}, a)$ is $1,-1$, $-1,1$, respectively. So $\sum_{a \in L_{r}} \mu_{L_{r}}(\hat{0}, a)=0$ unless $r=1,2$. For $r=1,2$, it can easily be checked that $\sum_{a \in L_{r}} \mu_{L_{r}}(\hat{0}, a)=1$ if $r=1$ and -1 if $r=2$. So if $r_{i} \geq 3$ for some $i$, then $\mu(\hat{0}, \hat{1})=0$. If each $r_{i} \in\{1,2\}$, then each index $i$ for which $r_{i}=2$ contributes a -1 to the product on the right-hand side of the previous equation. Thus $\mu(\hat{0}, \hat{1})=(-1)^{|S|+1}$.

For convenience, we introduce the following notations. Let $\Pi=\left\{312, \pi^{(1)}\right.$, $\left.\ldots, \pi^{(m)}\right\}$ where $\pi^{(j)}=\iota_{r_{j}}\left[\left(\pi_{1}^{(j)}\right)_{*}, \ldots,\left(\pi_{r_{j}}^{(j)}\right)_{*}\right]$. For $I=\left(i_{1}, \ldots, i_{m}\right)$, we define

$$
\underline{\Pi}_{I}=\left\{312, \underline{\pi^{(1)}}\left(i_{1}\right), \ldots, \underline{\pi^{(m)}}\left(i_{m}\right)\right\}
$$


and

$$
\bar{\Pi}_{I}=\left\{312, \overline{\pi^{(1)}}\left(i_{1}\right), \ldots, \overline{\pi^{(m)}}\left(i_{m}\right)\right\} .
$$

A generalization of Theorem 2.4 can be stated as the following.

Theorem 3.2. Suppose that the statistic st: $\mathfrak{S} \rightarrow \mathbb{N}$ satisfies the condition (†). Let $\Pi=\left\{312, \pi^{(1)}, \ldots, \pi^{(m)}\right\}$ where $\pi^{(i)}=\iota_{r_{i}}\left[\left(\pi_{1}^{(i)}\right)_{*}, \ldots,\left(\pi_{r_{i}}^{(i)}\right)_{*}\right]$. Then $F_{0}^{s t}(\Pi)=0$ if $\pi_{i}=\epsilon$ for some $i$ and 1 otherwise, and for $n \geq 1$ the stpolynomial $F_{n}^{\text {st }}(\Pi ; q)$ satisfies

$$
F_{n+1}^{s t}(\Pi ; q)=\sum_{k=0}^{n} q^{f(k, n-k)}\left[\sum_{S \subseteq[m]}(-1)^{|S|} \sum_{\substack{I=\left(i_{1}, \ldots, i_{m}\right): \\ 1 \leq i_{j} \leq r_{j}-\delta_{j}}} F_{k}^{s t}\left(\underline{\Pi}_{I}\right) \cdot F_{n-k}^{s t}\left(\bar{\Pi}_{I+\delta}\right)\right],
$$

where $\delta=\left(\delta_{1}, \ldots, \delta_{m}\right)$ with $\delta_{j}=1$ if $j \in S$ and 0 if $j \notin S$.

Proof. Recall that by Lemma 2.2 a permutation $\sigma=213\left[\sigma_{1}, 1, \sigma_{2}\right] \in \mathfrak{S}(312)$ avoids $\pi^{(j)}$ iff $\sigma$ satisfies the condition

$\left(C_{i}^{j}\right): \sigma_{1}$ avoids $\underline{\pi^{(j)}}(i)$ and $\sigma_{2}$ avoids $\overline{\pi^{(j)}}(i)$

for some $i \in\left[r_{j}\right]$. So $\sigma \in \mathfrak{S}(312)$ belongs to $\mathfrak{S}(\Pi)$ iff, for every $j$, there is an $i \in\left[r_{j}\right]$ for which $\sigma$ satisfies $\left(C_{i}^{j}\right)$. Fix $k$ and let $\mathfrak{S}_{n+1}^{k}(312)$ be as in the proof of Theorem 2.4. Let $A_{i}^{j}$ be the set of $\pi^{(j)}$-avoiding permutations in $\mathfrak{S}_{n+1}^{k}(312)$ satisfying the condition $\left(C_{i}^{j}\right)$. For $I=\left(i_{1}, \ldots, i_{m}\right) \in\left[r_{1}\right] \times\left[r_{2}\right] \times \cdots \times\left[r_{m}\right]$, we define the set $A_{I}$ to be

$$
A_{I}=A_{i_{1}, i_{2}, \ldots, i_{m}}:=A_{i_{1}}^{1} \cap A_{i_{2}}^{2} \cap A_{i_{m}}^{m} .
$$

So $\mathfrak{S}_{n+1}^{k}(\Pi)$ is the union

$$
\mathfrak{S}_{n+1}^{k}(\Pi)=\bigcup_{i_{1}, \ldots, i_{m}} A_{i_{1}, i_{2}, \ldots, i_{m}},
$$

where the union is taken over all $m$-tuples $I=\left(i_{1}, \ldots, i_{m}\right)$ in $\left[r_{1}\right] \times\left[r_{2}\right] \times$ $\cdots \times\left[r_{m}\right]$. Let $\hat{P}_{j}$ be the intersection poset of $A_{1}^{j}, \ldots, A_{r_{j}}^{j}$, and let $P_{j}$ be the poset $\hat{P}_{j} \backslash\left\{A^{j}\right\}$, where $A^{j}=A_{1}^{j} \cup \cdots \cup A_{r_{j}}^{j}$ is the unique maximum element of $\hat{P}_{j}$. Recall that $P_{j}$ is isomorphic to $L_{r_{j}}$. Let $P$ be the intersection poset of the $A_{I}$. The elements of $P$ are the unique maximal element $A=\mathfrak{S}_{n+1}^{k}(\Pi)$ and

$$
T=T^{1} \cap T^{2} \cap \cdots \cap T^{m},
$$

where each $T^{j}$ is an element of $P_{j}$. Thus $P$ is isomorphic to $L_{r_{1}} \times \cdots \times$ $L_{r_{m}} \cup\{\hat{1}\}$. For $S \subseteq[n]$, we say that an element $T \in P$ has type $S$ if 
$T^{j}=A_{i}^{j}$ for some $i$ when $j \notin S$ and $T^{j}=A_{i}^{j} \cap A_{i+1}^{j}$ for some $i$ when $j \in S$. Using Lemma 3.1, we know that the value of $\mu_{P}(T, A)$ where $T=$ $T^{1} \cap T^{2} \cap \cdots \cap T^{m} \neq A$ is

$$
\mu_{P}(T, A)= \begin{cases}(-1)^{|S|+1}, & \text { if } T \text { has type } S \\ 0, & \text { otherwise. }\end{cases}
$$

For $T \in P$, we define $g: P \rightarrow \mathbb{C}(x: x \in A)$ by $g(T)=\sum_{x \in T} x$, so that

$$
g(A)=\sum_{S \subseteq[n]}(-1)^{|S|} \sum_{T \text { has type } S} g(T)
$$

by the Möbius inversion formula.

Now, by the definition of type $S$, we have

$$
\sum_{T \text { has type } S} g(T)=\sum_{\substack{i_{1}, \ldots, i_{m}: \\ 1 \leq i_{j} \leq r_{j}-\delta_{j}}} g\left(\bigcap_{j \notin S} A_{i_{j}}^{j} \cap \bigcap_{j \in S}\left(A_{i_{j}}^{j} \cap A_{i_{j}+1}^{j}\right)\right),
$$

where $\delta_{j}=1$ if $j \in S$ and 0 if $j \notin S$. Recall that $\sigma \in A_{i_{j}}^{j}$ iff $\sigma_{1}$ avoids $\underline{\pi^{(j)}}\left(i_{j}\right)$ and $\sigma_{2}$ avoids $\overline{\pi^{(j)}}\left(i_{j}\right)$, and $\sigma \in A_{i_{j}}^{j} \cap A_{i_{j}+1}^{j}$ iff $\sigma_{1}$ avoids $\underline{\pi^{(j)}}\left(i_{j}\right)$ and $\sigma_{2}$ avoids $\overline{\pi^{(j)}}\left(i_{j}+1\right)$. Therefore, by mapping $\sigma \mapsto q^{\text {st }(\sigma)}$, we have

$$
\begin{aligned}
g\left(\bigcap_{j \notin S} A_{i_{j}}^{j} \cap \bigcap_{j \in S}\left(A_{i_{j}}^{j} \cap A_{i_{j}+1}^{j}\right)\right) & \mapsto q^{f(k, n-k)} F_{k}^{\mathrm{st}}\left(312, \underline{\pi^{(1)}}\left(i_{1}\right), \ldots, \underline{\pi^{(m)}}\left(i_{m}\right)\right) \\
& \cdot F_{n-k}^{\mathrm{st}}\left(312, \overline{\pi^{(1)}}\left(i_{1}+\delta_{1}\right), \ldots, \overline{\pi^{(m)}}\left(i_{m}+\delta_{m}\right)\right) .
\end{aligned}
$$

Therefore,

$$
F_{n+1, k}^{\mathrm{st}}(\Pi ; q)=q^{f(k, n-k)}\left[\sum_{S \subseteq[m]}(-1)^{|S|} \sum_{\substack{i_{1}, \ldots, i_{m}: \\ 1 \leq i_{j} \leq r_{j}-\delta_{j}}} F_{k}^{\mathrm{st}}\left(\underline{\Pi}_{I}\right) \cdot F_{n-k}^{\mathrm{st}}\left(\bar{\Pi}_{I+\delta}\right)\right]
$$

and we are done.

Example 3.3. Let $\Pi=\left\{312, \pi^{(1)}, \pi^{(2)}\right\}$ where $\pi^{(1)}=2314=12\left[12_{*}, \epsilon_{*}\right]$ and $\pi^{(2)}=2143=12\left[1_{*}, 1_{*}\right]$. We want to compute $a_{n}=F_{n}^{\text {inv }}(\Pi)$ by using Theorem 3.2. There are four possibilities of $S \subseteq\{1,2\}$, and for each possibility the following table shows the appearing terms, where $\delta$ is again the Kronecker delta function. 


\begin{tabular}{|l|l|l|}
\hline$S=\emptyset:$ & $F_{k}^{\text {inv }}(312,12,1) \cdot F_{n-k}^{\text {inv }}(\Pi)$ & $=\delta_{0, k} \cdot a_{n-k}$ \\
& $F_{k}^{\text {inv }}(312,2314,1) \cdot F_{n-k}^{\text {inv }}(312,1,2143)$ & $=\delta_{0, k} \cdot \delta_{0, n-k}$ \\
& $F_{k}^{\text {inv }}(312,12,2143) \cdot F_{n-k}^{\text {inv }}(312,2314,21)$ & $=1$ \\
& $F_{k}^{\operatorname{inv}}(\Pi) \cdot F_{n-k}^{\text {inv }}(312,1,21)$ & $=\delta_{0, n-k} \cdot a_{k}$ \\
\hline$S=\{1\}:$ & $F_{k}^{\text {inv }}(312,12,1) \cdot F_{n-k}^{\text {inv }}(312,1,2143)$ & $=\delta_{0, k} \cdot \delta_{0, n-k}$ \\
& $F_{k}^{\text {inv }}(312,12,2143) \cdot F_{n-k}^{\text {inv }}(312,1,21)$ & $=\delta_{0, n-k}$ \\
\hline$S=\{2\}:$ & $F_{k}^{\text {inv }}(312,12,1) \cdot F_{n-k}^{\text {inv }}(312,2314,21)$ & $=\delta_{0, k}$ \\
& $F_{k}^{\text {inv }}(312,2314,1) \cdot F_{n-k}^{\text {inv }}(312,1,21)$ & $=\delta_{0, k} \cdot \delta_{0, n-k}$ \\
\hline$S=\{1,2\}:$ & $F_{k}^{\text {inv }}(312,21,1) \cdot F_{n-k}^{\text {inv }}(312,1,21)$ & $=\delta_{0, k} \cdot \delta_{0, n-k}$ \\
\hline
\end{tabular}

Hence the $a_{n}$ satisfy

$$
\begin{aligned}
a_{n+1} & =\sum_{q=0}^{n} q^{k}\left[\delta_{0, k} a_{n-k}+\delta_{0, n-k} \cdot a_{k}+1-\delta_{0, k}-\delta_{0, n-k}\right] \\
& =\left(1+q^{n}\right) a_{n}+\frac{1-q^{n+1}}{1-q}-\left(1+q^{n}\right) \\
& =\left(1+q^{n}\right) a_{n}+q\left(\frac{1-q^{n-1}}{1-q}\right) .
\end{aligned}
$$

In particular, by setting $q=1$ we get $a_{n+1}=2 a_{n}+n-1$ with $a_{0}=a_{1}=1$. Thus

$$
\left|\mathfrak{S}_{n}(312,2314,2143)\right|=2^{n}-n .
$$

The following construction of st-Wilf equivalences can be extracted from Theorem 3.2. A proof of this corollary uses a similar argument to that of Corollary 2.6 and is omitted here.

Corollary 3.4. Let st be a statistic satisfying $(\dagger)$. Let $\pi_{i}^{(j)}, \pi_{i}^{(j)}, 1 \leq j \leq$ $m, 1 \leq i \leq r_{m}$, be permutations such that

$$
\left\{312, \pi_{i_{1}}^{(1)}, \ldots, \pi_{i_{m}}^{(m)}\right\} \stackrel{s t}{=}\left\{312, \pi_{i_{1}}^{\prime(1)}, \ldots, \pi_{i_{m}}^{\prime(m)}\right\}
$$

for all m-tuples $I=\left(i_{1}, \ldots, i_{m}\right) \in\left[r_{1}\right] \times \cdots \times\left[r_{m}\right] . \operatorname{Set} \pi^{(j)}=\iota_{r}\left[\pi_{1 *}^{(j)}, \ldots, \pi_{r_{j} *}^{(j)}\right]$ and $\pi^{\prime(j)}=\iota_{r}\left[\pi_{1 *}^{\prime(j)}, \ldots, \pi_{r^{*}}^{\prime(j)}\right]$. Then $\Pi=\left\{312, \pi^{(1)}, \ldots, \pi^{(m)}\right\}$ and $\Pi^{\prime}=\{312$, $\left.\pi^{\prime(1)}, \ldots, \pi^{\prime(m)}\right\}$ are st-Wilf equivalent.

\section{References}

[1] T. Dokos, T. Dwyer, B. P. Johnson, B. E. Sagan, and K. Selsor (2012). Permutation patterns and statistics. Discrete Mathematics 312(8) 27602775. MR2945168 
[2] S. Elizalde (2011). Fixed points and excedances in restricted permutations. Electron. J. Combin. 18(2). MR2880679

[3] S. Elizalde (2004). Multiple pattern-avoidance with respect to fixed points and excedances. Electron. J. Combin. 11(1). MR2097317

[4] S. Elizalde and I. Pak (2004). Bijections for refined restricted permutations. J. Combin. Theory A 105(2) 207-219. MR2046080

[5] T. Mansour and A. Vainshtein (2001). Restricted 132-Avoiding Permutations. Adv. in Appl. Math. 26(3) 258-269. MR1818747

[6] A. Robertson, D. Saracino, and D. Zeilberger (2003). Refined Restricted Permutations. Annals of Combin. 6(3) 427-444. MR1980351

[7] B. E. Sagan and C. D. Savage (2012). Mahonian Pairs. J. Combin. Theory Ser. A 119(3) 526-545. MR2871748

[8] R. P. Stanley (2012). Enumerative Combinatorics Vol. 1, 2nd ed. Cambridge University Press, Cambridge. MR2868112

[9] J. West (1996). Generating trees and forbidden subsequences. Discrete Mathematics 157(1-3) 363-374. MR1417303

WutTisak TrongsiriWAT

Department of Mathematics

Massachusetts Institute of Technology

CAmbridge, MA 02139

USA

E-mail address: wuttisak@mit.edu

RECEIVED 29 JANUARY 2014 